\title{
On the propagation of diel signals in river networks using analytic solutions of flow equations
}

\author{
Morgan Fonley ${ }^{1,2,4}$, Ricardo Mantilla ${ }^{2,3}$, Scott J. Small ${ }^{2}$, and Rodica Curtu ${ }^{1}$ \\ ${ }^{1}$ Department of Mathematics, University of Iowa, Iowa City, Iowa, USA \\ ${ }^{2}$ Iowa Flood Center, IIHR Hydroscience and Engineering, University of Iowa, Iowa City, Iowa, USA \\ ${ }^{3}$ Department of Civil and Environmental Engineering, University of Iowa, Iowa City, Iowa, USA \\ ${ }^{4}$ Department of Mathematics, Alma College, Alma, Michigan, USA \\ Correspondence to: Morgan Fonley (fonleymr@alma.edu)
}

Received: 4 August 2015 - Published in Hydrol. Earth Syst. Sci. Discuss.: 24 August 2015

Revised: 17 April 2016 - Accepted: 17 June 2016 - Published: 19 July 2016

\begin{abstract}
Several authors have reported diel oscillations in streamflow records and have hypothesized that these oscillations are linked to evapotranspiration cycles in the watershed. The timing of oscillations in rivers, however, lags behind those of temperature and evapotranspiration in hillslopes. Two hypotheses have been put forth to explain the magnitude and timing of diel streamflow oscillations during low-flow conditions. The first suggests that delays between the peaks and troughs of streamflow and daily evapotranspiration are due to processes occurring in the soil as water moves toward the channels in the river network. The second posits that they are due to the propagation of the signal through the channels as water makes its way to the outlet of the basin. In this paper, we design and implement a theoretical model to test these hypotheses. We impose a baseflow signal entering the river network and use a linear transport equation to represent flow along the network. We develop analytic streamflow solutions for the case of uniform velocities in space over all river links. We then use our analytic solution to simulate streamflows along a self-similar river network for different flow velocities. Our results show that the amplitude and time delay of the streamflow solution are heavily influenced by transport in the river network. Moreover, our equations show that the geomorphology and topology of the river network play important roles in determining how amplitude and signal delay are reflected in streamflow signals. Finally, we have tested our theoretical formulation in the Dry Creek Experimental Watershed, where oscillations are clearly observed in streamflow records. We find that our solution produces streamflow values and fluctuations that are similar to those observed in the summer of 2011.
\end{abstract}

\section{Introduction}

Several authors have observed daily fluctuations in streamflow during periods of little or no rain (e.g., Bond et al., 2002; Graham et al., 2013; Gribovszki et al., 2008; Wondzell et al., 2007; Burt, 1979; Wondzell et al., 2010). These fluctuations have been attributed to various causes, especially to those driven by temperature, which undergo a daily cycle. Temperature affects several hydrological processes, including freeze/thaw rates, evaporation rates, viscosity of water, and transpiration rates. Although many factors may contribute to the daily cycle of streamflow, evapotranspiration seems to be dominant (Gribovszki et al., 2010). Hydrologic processes during periods of low flow are often overlooked in favor of investigating high flow and subsequent flood conditions. In spite of this, the consequences of hydrological processes during low flow remain critical in dictating land use and agricultural types (Mul et al., 2011); indicating the extent of global climate change (Arnell, 1998); and influencing the chemical makeup of water downstream (Stott and Burt, 1997) or the availability of water, which impacts fish populations and water treatment requirements (Burn et al., 2008). Therefore, establishing a clear theoretical link between daily oscillations in streamflow and daily temperature cycles is a fundamental research endeavor.

(Graham et al., 2013) have compiled a collection of suggested explanations for the behavior of oscillatory streamflow under dry conditions, including several hypotheses that suggest that water moves differently through the subsurface as the hillslope drains. On the other hand, the authors of (Wondzell et al., 2007) suggest that streamflow properties are the result of attenuation as flow propagates along the river 
link with decreasing velocity, which causes the flow to be increasingly "out of phase". During dry periods of low flow, the time between the maximum evapotranspiration and the minimum streamflow values has been of particular interest because this time delay grows as the dry season progresses, indicating that the response of the streamflow to the evapotranspiration forcing on water on the hillslope slows as more water is removed from the system. Sorting out which of these hypotheses is correct, or which processes are more dominant in determining daily streamflow oscillations, is a crucial step towards sorting out the connection between hydrological processes occurring in the basin.

While observing streamflow at the outlet of the Dry Creek Experimental Watershed in Idaho during July of 2011 (described in Sect. 2), we recognized the oscillatory pattern described by (Graham et al., 2013), (Wondzell et al., 2007), and others. We used information from the nearby weather station to plot outlet flow with temperature and discovered that the two are phase-locked. They are not in phase, which would imply synchronization of the two signals, but are instead offset from each other by an almost constant value during the month. The phase offset depicts the delays that occur through various means as described in (Graham et al., 2013). Additionally we have examined the streamflows at several other locations in the Dry Creek Experimental Watershed (see Sect. 2) and see that they, too, are phase-locked with each other, but again they are not perfectly in phase. This has led us to investigate more closely how streamflows in the river network combine and create the oscillatory patterns which have different phases at different locations, although they are undergoing the same forcing.

In this paper, we aim to design and implement a theoretical model to test the hypotheses that attribute time delays to flows in the river network. We start by assuming a particular baseflow pattern in each river link of a given river network. Then, we work with simplified routing equations that assume constant velocity and give rise to a linear transport equation that allows us to develop an analytic solution for the flow at any given point along the river network. By fixing the baseflow pattern, we remove the dependence of streamflow properties (e.g., amplitude and time delay) on soil processes. If the resulting streamflows along the river network exhibit oscillations with different time delays and amplitudes, then we conclude that the effects described in Wondzell et al. (2007) can be induced by different velocities in a river network, even in the absence of changes induced by groundwater processes. Importantly, our theoretical results include algorithmic calculations of the phase shifts caused by the river network and their relationship to stream velocity. The latter can be used to make predictions about streamflow at any point in the river network, in particular with respect to the time delay between maximum evapotranspiration and minimum streamflow.

The paper is structured in the following way: in Sect. 2 we describe the data that motivate this work. In Sect. 3, we describe the baseflow pattern and linear mass transport equa- tion to represent flow along the river network. In Sect. 3.1, we compute the analytic solution for partial streamflow at the outlet of a river network due to baseflow applied to one upstream hillslope. Then, in Sect. 3.2, we assemble the complete solution at the outlet when all hillslopes in the network experience the same baseflow and all links in the network have uniform properties. Section 4.2 through Sect. 4.3 describe multiple scenarios to test the effects of river network velocity on streamflow attributes and support the claim that decreasing amplitude and increasing time delay in the streamflow at the network outlet can be attributed to delays in the river network. Finally, Sect. 5 contains a short concluding discussion and ideas for future work.

\section{Motivation}

The Dry Creek Experimental Watershed in Idaho is a $28 \mathrm{~km}^{2}$ watershed where streamflow, soil moisture, and weather conditions are monitored at multiple locations ((McNamara, 2012)). At the outlet of the watershed (labeled Lower Gauge), diel signals can be seen in the streamflow during several of the years of observation during which dry conditions occurred. We have focused our observations on the summer of 2011. The watershed includes seven stream gauging stations. One such station (Treeline) reported no streamflow for the duration of our observations. The reporting gauges are named Bogus South (BS), Con1West (C1W), Con1East (C1E), Con2East (C2E), Con2Main (C2M), and Lower Gauge (LG). They drain upstream areas of $0.63,3.85$, $8.70,7.54,24.15$, and $27.12, \mathrm{~km}^{2}$, respectively.

The left panel of Fig. 1 shows the Dry Creek watershed and the location of the streamflow gauges that were used in this study. The right panel shows temperature (top) - which is assumed to drive the diel signal in the streamflow via evapotranspiration - along with the streamflows at the gauges $\mathrm{C} 2 \mathrm{E}$ (second) and C2M (third), which are near the center of the watershed, and streamflow at LG (bottom), at the outlet of the watershed. Although all streamflow signals oscillate with the same period, they are each offset from temperature by a particular constant phase so that they are phase-locked with temperature and, subsequently, with each other. For example, temperature has a peak at hour 42. The next streamflow peak at $\mathrm{C} 2 \mathrm{E}$ is at hour 54, while $\mathrm{C} 2 \mathrm{M}$ has a peak at hour 56, and LG has a peak at hour 55. These different peak times imply that the flow along the river network causes sufficient delay in the streamflow signal to be noteworthy, supporting the hypothesis of (Wondzell et al., 2007). To create more decisive support for the hypothesis, and to investigate the nature of the delays, we have developed a theoretical experiment, which is described and implemented in the next several sections. 

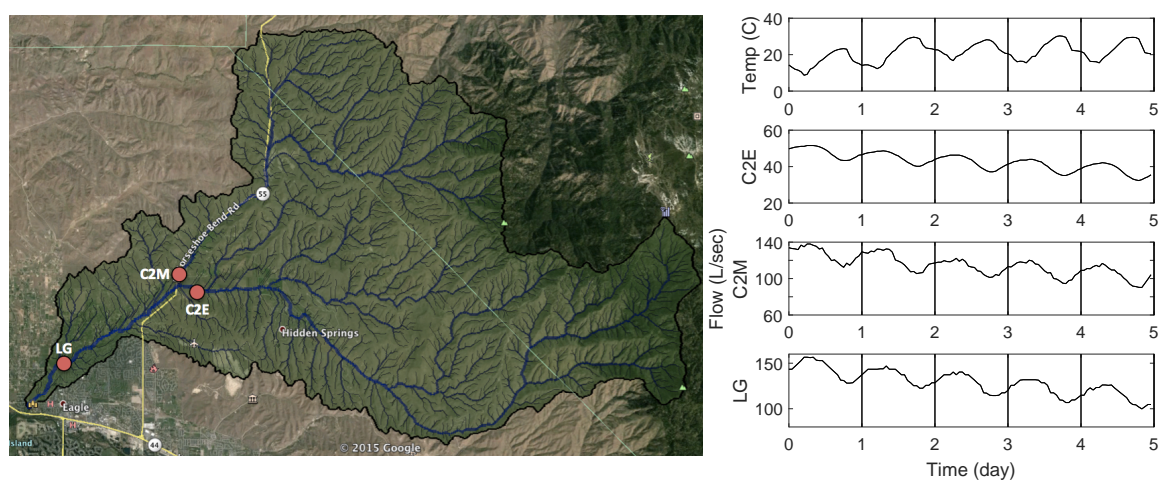

Figure 1. The left panel shows the Dry Creek watershed in Idaho. The right panel shows temperature (top), streamflow at gauge C2E near the center of the watershed (second), streamflow at gauge C2M (third), and streamflow at the outlet of the watershed (bottom). To demonstrate the delay in phases, a vertical line at the beginning of each day is included in each graph.

\section{Developing an analytic solution for streamflow based on river network geometry}

Let us now assume that the total subsurface runoff from each hillslope into a river link in a given river basin is oscillatory and its amplitude undergoes exponential decay (as seen for baseflow under dry conditions). Then, we define the runoff by the formula

$R(t)=B e^{-A t}+C e^{-A t} \sin (2 \pi v(t-\phi))$,

with $A, B, C$, and $v$ being positive parameters and $C<B$ to ensure that the baseflow takes only positive values. The phase shift $\phi$ represents an initial delay in observations due to water moving through the hillslope. In this paper, we apply the same baseflow pattern to all hillslopes on the river network beginning everywhere at an initial time $t=0$ (see the left panel of Fig. 2). Note that in this setup the runoff oscillations are supposed to be driven by evapotranspiration, which is synchronized over all hillslopes at the catchment scale. For this reason, synchronized timing of the forcing seems an acceptable hypothesis.

A sample baseflow pattern with parameter values $A=$ $0.003\left[\mathrm{~h}^{-1}\right], B=0.08\left[\mathrm{~L} \mathrm{~s}^{-1}\right], C=0.008\left[\mathrm{Ls}^{-1}\right]$, and $v=$ $\frac{1}{24}\left[\mathrm{~h}^{-1}\right]$ is illustrated in the right panel of Fig. 2. We chose the value of $v$ so that the frequency of the oscillations corresponds to a period of $24 \mathrm{~h}$, representing a diurnal signal. If we assume that the baseflow is linearly related to the amount of water in the soil, then $A$ corresponds to the linear rate of water movement through the soil.

In this paper, the streamflow at the outlet of a river link is defined as the solution to the system of ordinary differential equations, which has been derived from the mass conservation equation in the river links of the network, given by

$\frac{d q_{i}(t)}{d \mathrm{t}}=K\left(q_{i}\right)\left(R(t)+q_{i 1}(t)+q_{i 2}(t)-q_{i}(t)\right)$.

The inputs to the link come from runoff on adjacent hillslopes and from the streamflow of upstream tributary links.
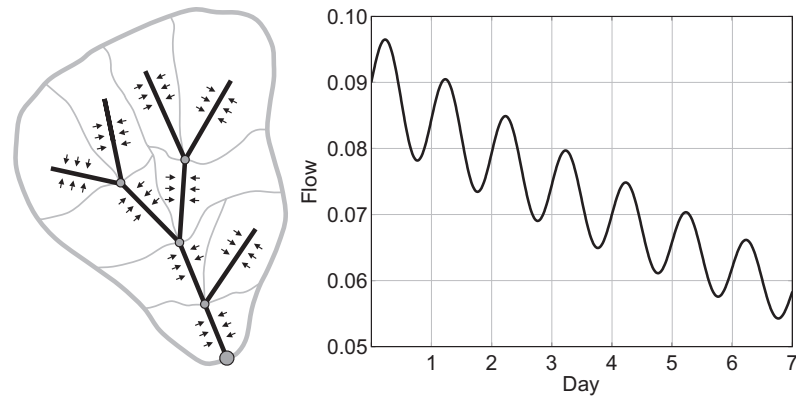

Figure 2. The left panel shows how runoff enters the river network as lateral flow from each hillslope to its adjacent link. The right panel shows a sample baseflow pattern given by Eq. (1) using $A=$ $0.003\left[\mathrm{~h}^{-1}\right], B=0.08\left[\mathrm{Ls}^{-1}\right], C=0.008\left[\mathrm{Ls}^{-1}\right], v=\frac{1}{24}\left[\mathrm{~h}^{-1}\right]$, and $\phi=0[\mathrm{~h}]$.

Therefore, the only method for water to exit the watershed is as streamflow at the outlet link. In the equation, $q_{i 1}$ and $q_{i 2}$ are the flows from the upstream tributary links. If a link $i$ has more than two tributaries at its upstream node, more terms can be added in Eq. (2), accordingly. For our calculation, we assume the function $K\left(q_{i}\right)$ to be constant, $K\left(q_{i}\right)=v_{i} / l$, where $v_{i}$ is the velocity of link $i$ and $l$ is the length of the link, which is assumed to be uniform over all links in the network (Mantilla et al., 2011). For simplicity, $K\left(q_{i}\right)$ will be called $k_{i}$.

To determine the streamflow at the river network outlet, we first consider the influence of runoff from a single hillslope and how that runoff signal propagates downstream; see Sect. 3.1 and Fig. 3. Then, in Sect. 3.2, we will assemble the information derived for all links of the river network into one comprehensive solution by applying the superposition principle. 


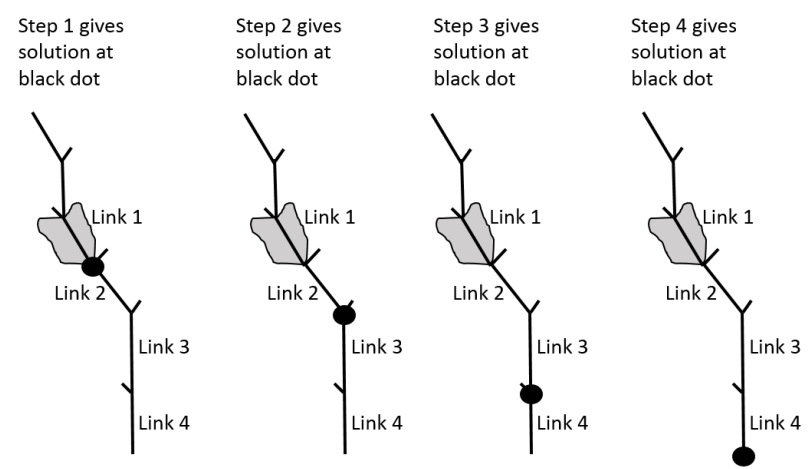

Figure 3. To determine the solution at any point, we consider runoff on only one hillslope (adjacent to link 1 in this case), and we trace the effects of that runoff downstream with no additional runoff from any subsequent hillslopes.

\subsection{Propagation of hillslope runoff signal on river networks with uniform velocity}

As mentioned above, we first apply runoff $R(t)$ to a given hillslope, denoted as "hillslope $a$ ", with adjacent river link 1. Because the transport equation for each link is linear, we can independently trace the runoff entering link 1 as it flows through the river network and then use superposition to combine the flows entering each river link. This would not be possible if the transport equation contained a nonlinear component. In the case of uniform velocities over the river network, the transport constant, $k_{i}$, is subsequently the same for all links in the network. In this subsection, it will be called $k$.

When the runoff entering link 1 has gone through one river link only ("step 1"; see Fig. 3), the flow $q_{1}$ at the outlet of link 1 is the solution to the differential equation

$\frac{d q_{1}(t)}{\mathrm{d} t}=k\left(B e^{-A t}+C e^{-A t} \sin (2 \pi v(t-\phi))-q_{1}(t)\right)$.

That is,

$q_{1}=\left(q_{1}(0)-\mathcal{J}_{1}+\mathcal{K}_{1} \sin (2 \pi v(\phi+\theta)) e^{-k t}+\left(\mathcal{J}_{1}\right.\right.$

$\left.+\mathcal{K}_{1} \sin (2 \pi v(t-\phi-\theta))\right) e^{-A t}$,

with $q_{1}(0)$ being the initial condition (at $t=0$ ) of the flow in link 1 and $\mathcal{K}_{1}, \mathcal{J}_{1}$, and $\theta$ defined by

$\mathcal{K}_{1}=\frac{C k}{\sqrt{(k-A)^{2}+4 \pi^{2} v^{2}}}$,

$\mathcal{J}_{1}=\frac{B k}{k-A}$

and

$$
\begin{aligned}
& \sin (2 \pi \nu \theta)=\frac{2 \pi \nu}{\sqrt{(k-A)^{2}+4 \pi^{2} v^{2}}}, \\
& \cos (2 \pi \nu \theta)=\frac{k-A}{\sqrt{(k-A)^{2}+4 \pi^{2} \nu^{2}}} .
\end{aligned}
$$

Note that $\theta \in\left(0, \frac{1}{4 v}\right)$ is the resulting time delay for the fluctuating pattern $q_{1}(t)$ of frequency $v$ compared to the input signal $R(t)$.

At step 2, when the runoff has traversed two river links, we need to compute $q_{2}(t)$ by taking into account the solution $q_{1}(t)$ from step 1 (see Fig. 3, second panel). Since we assumed for the moment that $q_{1}(t)$ has been transmitted downstream via the next link (link 2), with no additional runoff, the streamflow at the end of link 2 is given by

$$
\begin{aligned}
q_{2} & =\left[\left(q_{2}(0)-\mathcal{J}_{2}+\mathcal{K}_{2} \sin \left(2 \pi v \theta_{2}\right)\right)\right. \\
& \left.+k t\left(q_{1}(0)-\mathcal{J}_{1}+\mathcal{K}_{1} \sin \left(2 \pi v \theta_{1}\right)\right)\right] e^{-k t} \\
& +\left(\mathcal{J}_{2}+\mathcal{K}_{2} \sin \left(2 \pi v\left(t-\theta_{2}\right)\right)\right) e^{-A t},
\end{aligned}
$$

with $\theta_{1}=\phi+\theta, \theta_{2}=\phi+2 \theta$, and

$$
\begin{aligned}
\mathcal{K}_{2} & =\frac{C k^{2}}{(k-A)^{2}+4 \pi^{2} v^{2}} \\
\mathcal{J}_{2} & =\frac{B k^{2}}{(k-A)^{2}} .
\end{aligned}
$$

By mathematical induction, we then compute the solution $q_{n}(t), n \geq 1$ of flow measured downstream at the exit from link $n$. This takes the form

$q_{n}(t)=e^{-A t}\left[\mathcal{J}_{n}+\mathcal{K}_{n} \sin \left(2 \pi v\left(t-\theta_{n}\right)\right)\right]+e^{-k t} \sum_{j=0}^{n-1} \mathcal{L}_{n-j} \frac{(k t)^{j}}{j !}$

with coefficients

$$
\begin{array}{rlrl}
\mathcal{K}_{n} & =C \prod_{j=1}^{n} \frac{k}{\sqrt{(k-A)^{2}+4 \pi^{2} v^{2}}} & \\
& =C\left(\frac{k}{\sqrt{(k-A)^{2}+4 \pi^{2} v^{2}}}\right)^{n}, & & n \geq 1 \\
\mathcal{J}_{n} & =B \prod_{j=1}^{n} \frac{k}{k-A}=B\left(\frac{k}{k-A}\right)^{n}, & & n \geq 1 \\
\theta_{n} & =\phi+\sum_{i=1}^{n} \theta=n \theta, & & n \geq 1
\end{array}
$$

and

$\mathcal{L}_{j}=q_{j}(0)-\mathcal{J}_{j}+\mathcal{K}_{j} \sin \left(2 \pi v \theta_{j}\right) \quad, j=1,2, \ldots n$.

Here, $q_{j}(0)$ represents the initial condition for the flow in link $j$. For clarity, we included the details of this algorithmic proof in Appendix A.

\subsection{Assembling the complete solution for streamflow at the outlet}

The goal of this section is to determine the equation for the streamflow at a given point of calculation along the river network, in particular at the network outlet. We take the parameters representing properties of each river link to be uniform 


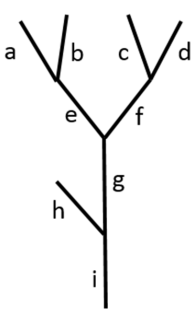

Figure 4. A small sample network to describe how total streamflow is computed.

over all links in the network (i.e., same parameter $k$ ) so that the influence of two links that are equidistant (topologically speaking) from the outlet will be the same. The solution determined in Sect. 3.1, however, shows only the partial contribution of link $i$ to the streamflow, as it propagates downstream without considering any additional runoff. Therefore, in order to determine the complete streamflow solution, one must sum the overall contributions from runoff on each upstream link. This can be done if the topological representation of the river network is known or if the topological width function upstream of the outlet is used. The width function for a given link $i$ and distance $n$ (denoted $W_{n}^{(i)}$ ) is an integer representing the number of river links of topological distance $n$ upstream of link $i$, where $W_{1}^{(i)}=1$ and corresponds to link $i$ itself. For a fixed location in the river network, the width function can be written as a vector whose length is the diameter (i.e., the longest path) upstream of link $i$. The network depicted in Fig. 4 further illustrates this process.

First, we will focus on the outlet of link $a$ (before the streamflow from $a$ combines with that of link $b$ ); see Fig. 4 . We recognize one link upstream of this point: link $a$. Then, the only contribution to the streamflow at this point is from the runoff to link $a$ that has traversed one link. The width function at this point has only one element, and there is only one link of distance 1 , so the width function, a onedimensional vector, is given by $W^{(a)}=[1]$, and the streamflow is simply

$q_{a}=1 \times q_{1}=q_{1}=\mathcal{L}_{1} e^{-k t}+e^{-A t}\left[\mathcal{J}_{1}+\mathcal{K}_{1} \sin \left(2 \pi v\left(t-\theta_{1}\right)\right)\right]$.

On the other hand, if we compute streamflow at the outlet of link $e$ (prior to joining link $f$; see Fig. 4), we have one link of topological distance 1 (link $e$ ) and two links of topological distance 2 (links $a$ and $b$ ). Then, the width function is given by the vector $W^{(e)}=\left[\begin{array}{ll}1 & 2\end{array}\right]$. This means that the runoff from link $e$ has only traversed one link to get to the outlet, but the runoff from either of the links $a$ or $b$ has traversed two links. The total flow at the outlet of link $e$ is

$q_{e}=1 \times q_{1}+2 \times q_{2}=q_{1}+2 q_{2}$.

After applying the formulas for $q_{1}$ and $q_{2}$, similar terms can be collected in the following way:

$q_{e}=\mathcal{L}_{1} e^{-k t}+e^{-A t}\left[\mathcal{J}_{1}+\mathcal{K}_{1} \sin \left(2 \pi v\left(t-\theta_{1}\right)\right)\right]$

$$
\begin{aligned}
& +2\left[\mathcal{L}_{2}+k t \mathcal{L}_{1}\right] e^{-k t}+2\left[\mathcal{J}_{2}+\mathcal{K}_{2} \sin \left(2 \pi v\left(t-\theta_{2}\right)\right)\right] e^{-A t} \\
& =e^{-A t}\left(\mathcal{J}_{1}+2 \mathcal{J}_{2}+\mathcal{K}_{1} \sin \left(2 \pi v\left(t-\theta_{1}\right)\right)\right. \\
& \left.+2 \mathcal{K}_{2} \sin \left(2 \pi v\left(t-\theta_{2}\right)\right)\right)+e^{-k t}\left(\mathcal{L}_{1}+2\left[\mathcal{L}_{2}+k t \mathcal{L}_{1}\right]\right) .
\end{aligned}
$$

To complete this example, let us now consider the width function at the outlet of the network in Fig. 4, which is $W^{(i)}=\left[\begin{array}{llll}1 & 2 & 2 & 4\end{array}\right]$. The first element of $W^{(i)}$ corresponds to link $i$; the second element $\left(W_{2}^{(i)}=2\right)$ corresponds to links $g$ and $h$; the third element $\left(W_{3}^{(i)}=2\right)$ corresponds to links $e$ and $f$; and the last component $\left(W_{4}^{(i)}=4\right)$ corresponds to links $a, b, c$, and $d$. The diameter of this network is $D_{i}=$ length $\left(W^{(i)}\right)=4$. Note that the total number of links in the network is also the sum of the elements of the width function, since each link has a corresponding distance from the outlet. For this, we can use the notation $\left|W^{(i)}\right|=\sum_{n=1}^{D_{i}} W_{n}^{(i)}=9$. For more details about the width function, see Mantilla et al. (2011) and Rodriguez-Iturbe and Rinaldo (2001). The flow at the outlet of link $i$ is

$$
\begin{aligned}
q_{i} & =1 \times q_{1}+2 \times q_{2}+2 \times q_{3}+4 \times q_{4}=\sum_{n=1}^{D_{i}} W_{n}^{(i)} q_{n} . \\
& =e^{-A t}\left(\mathcal{J}_{1}+2 \mathcal{J}_{2}+2 \mathcal{J}_{3}+4 \mathcal{J}_{4}\right)+e^{-A t}\left(\mathcal{K}_{1} \sin \left(2 \pi v\left(t-\theta_{1}\right)\right)\right. \\
& +2 \mathcal{K}_{2} \sin \left(2 \pi v\left(t-\theta_{2}\right)\right)+2 \mathcal{K}_{3} \sin \left(2 \pi v\left(t-\theta_{3}\right)\right. \\
& \left.+4 \mathcal{K}_{4} \sin \left(2 \pi v\left(t-\theta_{4}\right)\right)\right)+e^{-k t}\left(\mathcal{L}_{1}+2\left[\mathcal{L}_{2}+k t \mathcal{L}_{1}\right]\right. \\
& +2\left[\mathcal{L}_{3}+k t \mathcal{L}_{2}+\frac{(k t)^{2} \mathcal{L}_{1}}{2 !}\right] \\
& \left.+4\left[\mathcal{L}_{4}+k t \mathcal{L}_{3}+\frac{(k t)^{2} \mathcal{L}_{2}}{2 !}+\frac{(k t)^{3} \mathcal{L}_{1}}{3 !}\right]\right) .
\end{aligned}
$$

For a general network whose width function is given by $W^{(i)}$, the solution can be rearranged as in Eqs. (12) and (14) to get the complete solution for streamflow at the outlet $i$. Assuming that $D_{i}$ is the diameter of the network upstream of link $i$, the solution at the outlet $i$ is

$$
\begin{aligned}
q_{i} & =e^{-A t} \sum_{n=1}^{D_{i}} W_{n}^{(i)}\left[\mathcal{J}_{n}+\mathcal{K}_{n} \sin \left(2 \pi v\left(t-\theta_{n}\right)\right)\right] \\
& +e^{-k t} \sum_{n=1}^{D_{i}} W_{n}^{(i)} \sum_{j=0}^{n-1} \mathcal{L}_{n-j} \frac{(k t)^{j}}{j !} .
\end{aligned}
$$

The first term in Eq. (15) represents the propagation of the runoff signal from each hillslope, while the second term is a result of the initial conditions coming from runoff and flow in the network. This distinction is evidenced by the rate of decay of either exponential function. The first term has a rate of decay depending upon A and represents the decay of runoff entering the channel. The second term, conversely, has a decay rate dependent only upon $k$, which describes the rate of water movement through each river link.

To thoroughly interpret the components of Eq. (15), we again contemplate the physical processes being represented 
and use the expected parameter values to discuss the mathematical solution. First, $k$ and $A$ are both positive because they represent rates of water movement along the river link and through the soil, respectively. Since water will move much more quickly along the river link, which offers less resistance than soil, $A$ is significantly less than $k$, so that $\frac{k}{k-A}$ has a value slightly greater than 1 . Then, $\mathcal{J}_{j}>B$ for any value of $j$. Furthermore, the value of $2 \pi v$ is fixed and is typically greater than $k$, which means that $\frac{k}{\sqrt{(k-A)^{2}+4 \pi^{2} v^{2}}}<1$, so that $\mathcal{K}_{j}<C$ for all $j$. This means that each component $\left[\mathcal{J}_{n}+\mathcal{K}_{n} \sin \left(2 \pi v\left(t-\theta_{n}\right)\right)\right]$ of the solution at the outlet shows a decrease in the amplitude of the fluctuations $\left(\mathcal{K}_{n}<C\right)$ while increasing its average value when compared with the runoff function $\left(\mathcal{J}_{n}>B\right)$.

In the limiting case of $A=0$, the runoff at each hillslope would be a sinusoidal wave of amplitude $C$ and average value $B$ taking the form $R=B+C \sin (2 \pi v t)$. Then, the solution at the outlet becomes

$$
\begin{aligned}
q_{i} & =\sum_{n=1}^{D_{i}} W_{n}^{(i)}\left[\mathcal{J}_{n}+\mathcal{K}_{n} \sin \left(2 \pi v\left(t-\theta_{n}\right)\right)\right] \\
& +e^{-k t} \sum_{n=1}^{D_{i}} W_{n}^{(i)} \sum_{j=0}^{n-1} \mathcal{L}_{n-j} \frac{(k t)^{j}}{j !}
\end{aligned}
$$

where $\mathcal{K}_{n}, \mathcal{J}_{n}$, and $\theta$ are defined by $\mathcal{K}_{n}=C \prod_{i=1}^{n} \frac{k}{\sqrt{k^{2}+4 \pi^{2} v^{2}}}$, $\mathcal{J}_{n}=B$, and $\sin (2 \pi \nu \theta)=\frac{2 \pi \nu}{\sqrt{k^{2}+2 \pi^{2} \nu^{2}}}$ and $\cos (2 \pi \nu \theta)=$ $\frac{k}{\sqrt{k^{2}+2 \pi^{2} \nu^{2}}}$.

It is apparent that the second sum of Eq. (16) that includes exponential decay at the rate of water movement through the river link is the transient term. The first sum of Eq. (16) is the asymptotic solution and includes the sum of constant terms from each hillslope and the sum of amplitudes of the sine waves from each hillslope. Following a similar approach in the case of $A>0$ and using the fact that $A \ll k$, we again find that the second term in Eq. (15) decays much faster; consequently, $e^{-A t} \sum_{n=1}^{D_{i}} W_{n}^{(i)}\left[\mathcal{J}_{n}+\mathcal{K}_{n} \sin \left(2 \pi v\left(t-\theta_{n}\right)\right)\right]$ can be interpreted as being the asymptotic solution of $q_{i}$. Due to interference from sinusoidal waves that can be in or out of phase, the amplitude of the asymptotic solution in $q_{i}$ can change depending on the phase shift. We investigate this dependence in Sect. 4.

\section{Results}

\subsection{Testing design: examining the effects of velocity on streamflow amplitude and time delay downstream}

In order to test the competing hypotheses by Wondzell et al. (2007) and those presented in Graham et al. (2013), we will demonstrate the amplification and damping of the oscillatory streamflow signal that are caused by superposition. We consider a sample network and compute the streamflow solution at different locations in the river network when the velocity and its corresponding time delay are varied. We will consider both the uniform (with $v_{i}=v$ for all links $i$ ) and the variable velocity cases.

We compute the streamflow solution for the MandelbrotVicsek tree of magnitude 14, as shown in Fig. 5. The Mandelbrot-Vicsek tree is self-similar (Mandelbrot and Vicsek, 1989) and has been used to demonstrate hydrologic properties at different scales (e.g., Mantilla et al., 2006; Peckham, 1995). In this figure, the label next to each link represents the magnitude of the link, which describes the scale of the link and is determined by the sum of the magnitudes of the two immediate upstream "parent" links where external links have magnitude 1 . The constant parameter values used in this example are $A=1.2 \times 10^{-4}\left[\mathrm{~h}^{-1}\right], B=0.08$ $\left[\mathrm{Ls}^{-1}\right], C=0.008\left[\mathrm{~L} \mathrm{sec}^{-1}\right], q_{0}=0.08\left[\mathrm{~L} \mathrm{sec}^{-1}\right]$, and $v=$ $\frac{1}{24}\left[\mathrm{~h}^{-1}\right]$ and are uniform over each link in the network. To test the effects of superposition on streamflow, we will simulate streamflow for different transport constants $k$. Figure 6 shows the simulation runoff pattern (top) along with the sample streamflow solution at the outlet of the network in the uniform case (bottom). To distinguish among the different simulations, we will narrow our view to a few oscillations, which are highlighted by a box in the panels in Fig. 6 .

\subsection{Uniform velocity over the river network}

In the case of uniform velocities, the streamflow at the outlet is given by the solution to Eq. (15). The time delay depends upon parameters that have physically based values (see Eq. 6), so a realistic range for the time delay and phase shift can be found. These parameters, $k$ and $A$, are incorporated in other parts of the solution (see Eq. 8). Therefore, changing their values impacts the solution in more ways than just the superposition of sinusoidal functions. The physical value represented by $A$ is expected to remain constant for a given region. On the other hand, $k$ represents the inverse of the residence time in each river link and is not necessarily uniform or fixed.

Recall that $k$ is given by $\frac{v}{l}$, where $v$ is the stream velocity and $l$ is the stream length. The length of each river link in a real river network would be different, as would the velocity. In addition, the velocity may change over time, since velocity increases with flow. Consequently, the realistic value of $k$ is expected to be different for each link in the network, and the uncertainty of $k$ is a possible source for different time delays and phase shifts.

While the effect of varying $k$ is not limited to the time delay, the value of $k$ also affects $\mathcal{K}_{n}$ and $\mathcal{J}_{n}$ (see Eq. 8). Note that the coefficient $\mathcal{J}_{n}$ determines the average value of the streamflow solutions, while the coefficient $\mathcal{K}_{n}$ determines the amplitude of the oscillation in each step of the streamflow solution (see Eq. 15, first term). Changing $k$, then, impacts the amplitude downstream more significantly than simply altering the time delay and subsequent phase shift. 


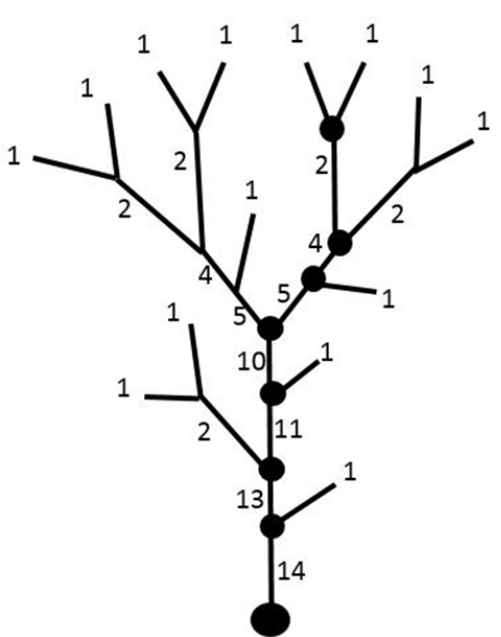

Figure 5. The Mandelbrot-Vicsek tree of magnitude 14. The magnitude of each link is written next to the link. One link of each magnitude is distinguished by the dots along the network.
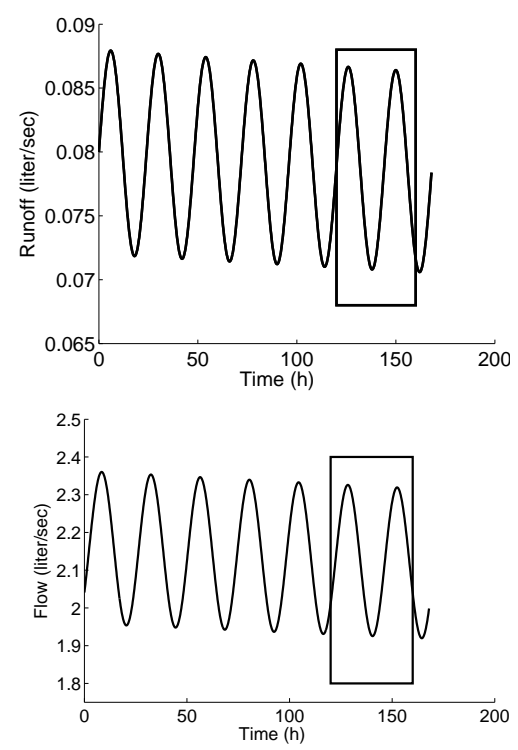

Figure 6. Sample runoff pattern (top) and resulting streamflow solution at the outlet in the uniform case (bottom) for $k=\frac{v}{T}$ To examine the oscillations more closely for different velocities, we will focus on a small section of the solution (highlighted by a box in each panel).

The results of simulating streamflow in the MandelbrotViscek tree using different values of $k$ can be found in Fig. 7. The values of $k$ used in simulations are $0.38,0.7,1.02,1.34$, $1.66,1.98$, and 2.30 with resultant time delays of $2.30,1.36$, $0.95,0.73,0.59,0.5$, and $0.43 \mathrm{~h}$. The corresponding graph solutions from Fig. 7 are drawn in the following colors: black, blue, green, cyan, orange, red, and purple, respectively. Each panel in Fig. 7 represents the solution at a different location along the network (refer to Fig. 5 for sample locations).
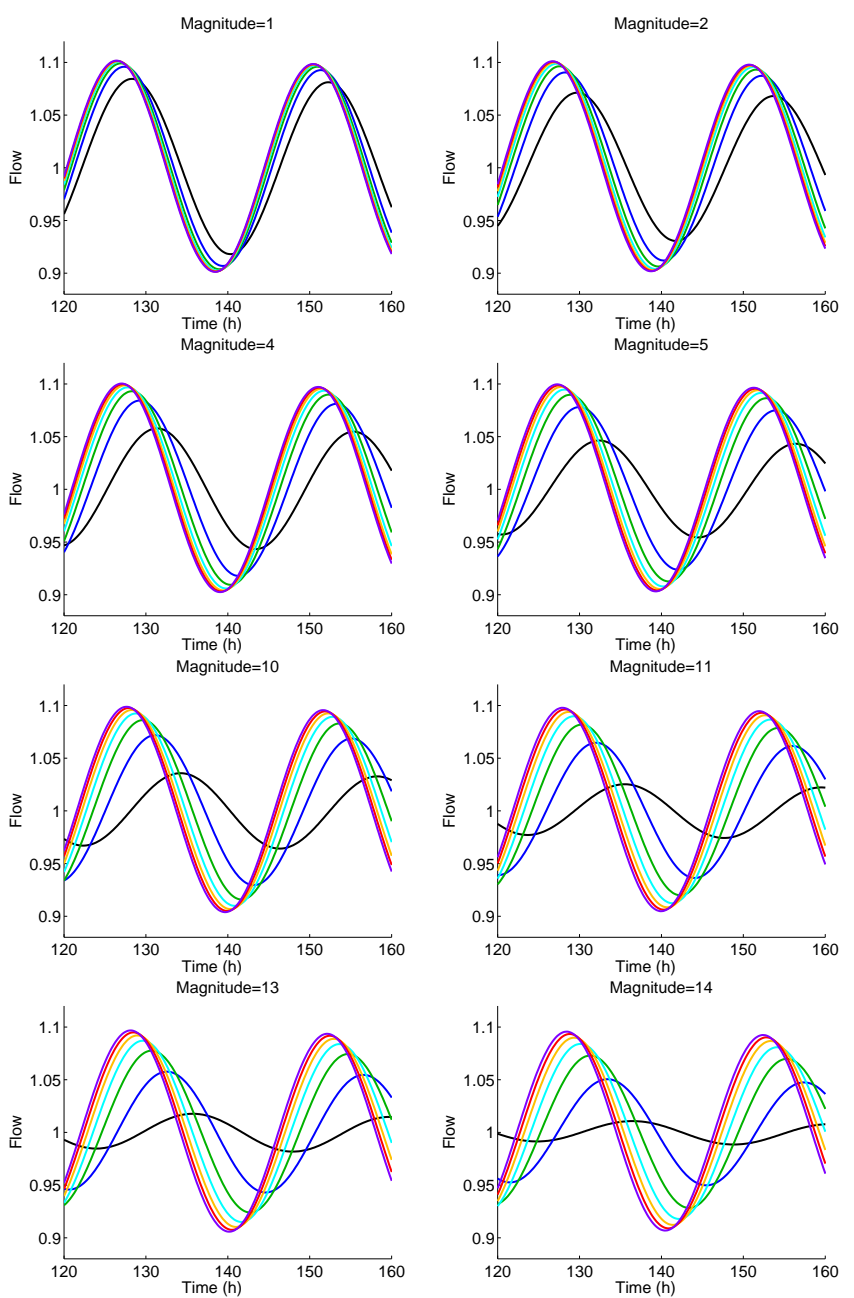

Figure 7. Flows at the outlet of each magnitude link using different $k$ in each river simulation. The $k$ values (with units of $\mathrm{h}^{-1}$ ) are $0.38,0.7,1.02,1.34,1.66,1.98$, and 2.30 and are colored black, blue, green, cyan, orange, red, and purple, respectively. The flows are normalized about the average flow.

We chose the timing of the plots so that a cyclic pseudoequilibrium has been reached and the effects of time delay can be distinguished. For comparison among the different locations, we have normalized the flows about the average flow. The average flows at a link of each magnitude are plotted in Fig. 8; as expected, the values depend upon the number of links upstream, which is related to the magnitude of the link.

From Fig. 7, we see that the magnitude of the oscillations can be significantly decreased as velocity and $k$ decrease, because this represents a volume of water spending more time in any one link. This causes a greater time delay, which means that two links will combine their flows out of phase, and superposition dictates that the amplitude of the resulting oscillations is decreased. Furthermore, a lower velocity leads to significant attenuation of the streamflow along each link in the network. The greatest amplitudes occur when the veloc- 


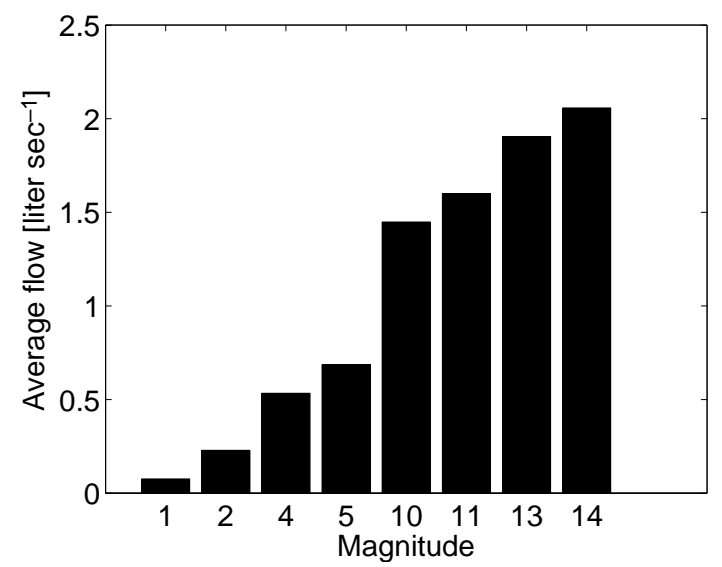

Figure 8. Average flows at different locations along the Mandelbrot-Vicsek tree.

ity is highest, which moves a volume of water very quickly through each link and leads to very little loss of streamflow intensity. Notice also that the timing of the peak streamflow is increasingly delayed as velocity slows (see Fig. 7 for $k=0.38,1.02$, and 1.66 , for example). This can explain the increasing time delay that has been observed between maximum evapotranspiration and minimum streamflow as the dry season progresses. These results also indicate that the time delay increases continuously as the velocity decreases continuously over time so that the time delay can be predictable depending upon stream velocity.

At the link of magnitude 1, the phase shift has little influence on the amplitude and only has an influence on the timing of the wave. At the outlet of a magnitude-2 link, the two upstream links are "in phase", meaning they have the same time delay as each other since they are the same topological distance from the point at which we compute streamflow. Therefore, these two will exhibit constructive interference. When they are combined with the downstream link, however, the different values of phase shift can result in constructive or destructive interference, although they never completely destroy the oscillations. The phase shift that produces the maximum streamflow is zero because this represents the face that all three streamflows that feed into this outlet are completely in phase.

As we examine the streamflows in links with greater magnitude, the shape of the network (described by the width function) becomes important because the flows from all links of a given distance will reach the outlet at the same time. Being out of phase with links of other distances can cause some reduction in the amplitude of the streamflow oscillations, but the oscillations will not be completely destroyed.

\subsection{Propagation of oscillations on a real network}

In this section, we apply the analytic streamflow solution for uniform conditions to the river network of the Dry Creek river basin to study the effects of scale on streamflow amplitude and timing. In the previous section, we also examined the flow at different scales (see Fig. 7) but with an emphasis on different $k$ values. The time range in Fig. 7 has been decreased, and the flows have been normalized about their average value to exaggerate the effects of changing the $k$ value. Consider the blue line in all panels of Fig. 7 corresponding to a $k$ value of $0.7 \mathrm{~h}^{-1}$. Streamflow at a larger scale (magnitude) is influenced by a greater number of upstream links. Hence, superposition effects among those upstream links are stronger, and we see two resulting attributes in the streamflow properties: reduction in the streamflow amplitude and greater time delay to the peak. We now consider a larger, more realistic river network and expect to see similar results.

In our theoretical examples, we assumed the length of each link to be uniform over the river network, so that changes in velocity directly correspond to changes in the transport constant $k$. Realistic network parameters include variable link length, so we vary the velocity of each link accordingly in order to maintain a uniform $k$ value and apply the solution developed in Sect. 3.

For a comparison with available data, we revisit information from the Dry Creek Experimental Watershed in Idaho. Using streamflow data from LG, the gauge nearest the watershed outlet along with topological data retrieved using the program CUENCAS proposed in (Mantilla and Gupta, 2005), we can compare the diel flows observed in 2011 with the solution method used in Sect. 3. Specifically, the solution to describe streamflow at the outlet, given in Eq. (16), can be fitted to the observed streamflow to find parameter values $\mathrm{A}, \mathrm{B}$, and $\mathrm{C}$ that uniquely describe baseflow exiting each hillslope. The assumption inherent in this solution is that the river links are all uniform, which is an unrealistic but necessary simplification to develop this explicit solution. The top left panel of Fig. 9 depicts the observed streamflow from July of 2011 along with our approximated solution that was found by fitting the data to Eq. (16) using MATLAB. The resulting parameter values are

$$
\begin{array}{ll}
A=1.85 \times 10^{-3} & {\left[\mathrm{~h}^{-1}\right]} \\
B=0.239 & {\left[\mathrm{~L} \mathrm{~s}^{-1}\right]} \\
C=3.27 \times 10^{-2} & {\left[\mathrm{~L} \mathrm{~s}^{-1}\right]} \\
\phi=3.97 & {[\mathrm{~h}]} \\
k=5.61 & {\left[\mathrm{~h}^{-1}\right] .}
\end{array}
$$

For perspective, this $k$ value corresponds to an average stream velocity of $0.38 \mathrm{~m} \mathrm{~s}^{-1}$.

Using the observed streamflow time series at several upstream gauges in the Dry Creek watershed, we can test our analytic solution with the parameters determined above. If we treat these locations as the outlets of smaller embedded watersheds, we can again apply Eq. (16) using the same parameter values which will yield our solution at points along the river network. The center and lower panels of Fig. 9 depict the observed streamflows and the streamflows computed using Eq. (16) at gauges $\mathrm{C} 2 \mathrm{M}$ and $\mathrm{C} 2 \mathrm{E}$, respectively. 

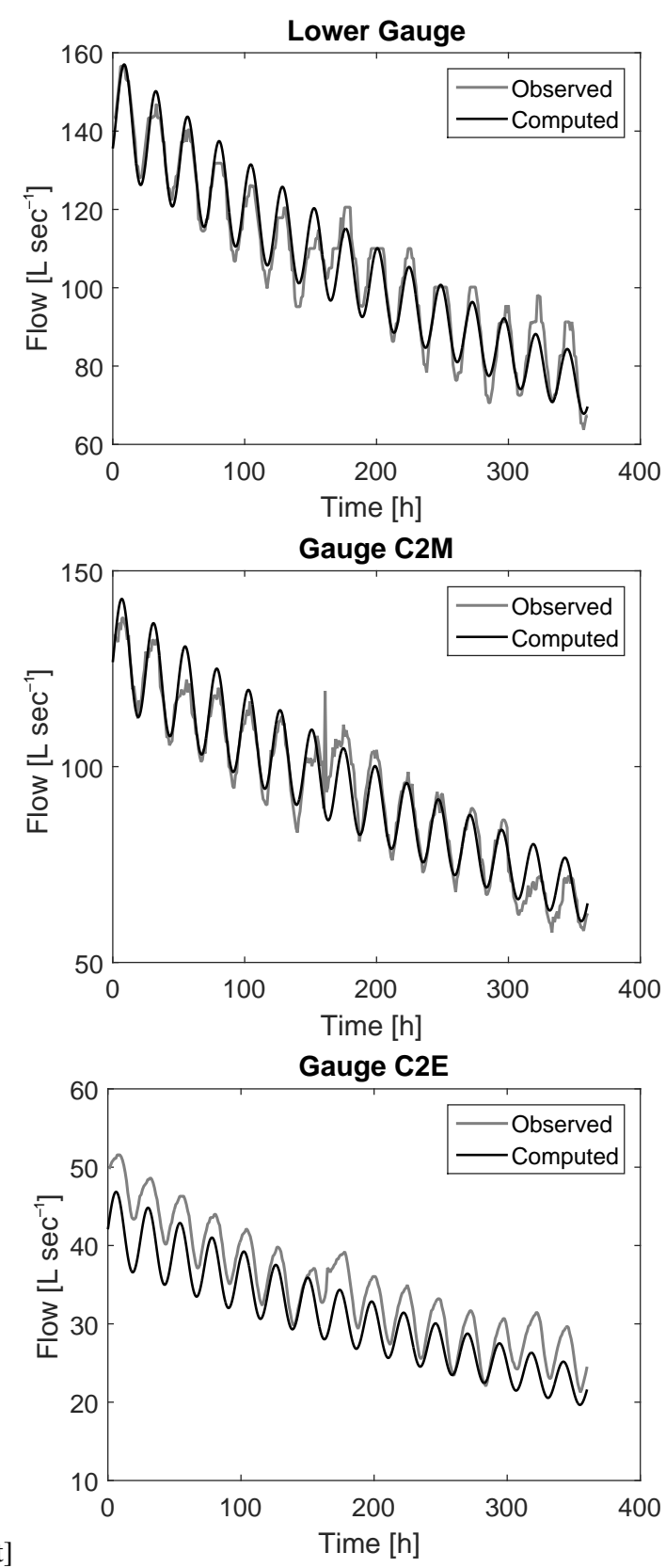

Figure 9. Observed streamflow and streamflow fitted using Eq. (16) at the outlet of the Dry Creek Experimental Watershed (top) and at two stream gauges upstream in the watershed: Con2Main (center) and Con2East (bottom).

Although the predicted streamflow given by our solution does not fit the data as well for $\mathrm{C} 2 \mathrm{E}$ as it does for $\mathrm{C} 2 \mathrm{M}$, we can see that the magnitude of our predicted streamflow is very close to observed streamflow at either location. Furthermore, the timing of the oscillations is nearly identical for both $\mathrm{C} 2 \mathrm{M}$ and $\mathrm{C} 2 \mathrm{E}$. In the presence of heterogeneity on the hillslope and along the river network, we must be flexible about the amount of data we can reasonably expect to fit well.
For example, we show in Appendix B that the data are more noisy at other gauges in the Dry Creek watershed where we compare observed streamflow data to our streamflow solution.

Because our solution fits the data reasonably well at several locations along the river network where runoff is uniformly enforced, we can be assured of the internal validity of using a solution such as that given in Eq. (16). Furthermore, because our solution describes superposition among all the oscillating runoff signals entering the network, and the simulation results are close to those observed, we can conclude that streamflow relies heavily on superposition from upstream in the river network as suggested in (Wondzell et al., 2007).

\section{Conclusions and future work}

Observations of oscillatory streamflow during low-flow conditions have highlighted the magnitude and time delay caused by the diel signal that represents evapotranspiration. Several current hypotheses suggest that the properties of the oscillatory streamflow signal can be attributed to different methods of water movement through the subsurface, although another hypothesis suggests that flow along the river determines the timing and amplitude of oscillations. In this paper, we provide evidence to support the latter argument.

First, we select a mathematical function according to streamflow observations at the catchment scale to represent baseflow patterns at the hillslope scale. The selected baseflow pattern is applied as input to a linear transport equation for all links in a river network that are assumed to have uniform properties and parameter values. For this uniform situation, we develop an analytic solution to represent streamflow at any point in a river network. We compute the solution by separately determining the partial streamflow at the outlet from each river link and then taking the sum over all river links in the river network. In order to include the geomorphology of the river network, we use the width function to compute the complete streamflow solution. We have also extended the streamflow solution to include nonuniform links in the river network.

The solution for streamflow contains a collection of sine functions, each of which exhibits a phase shift determined by the topological distance of the corresponding hillslope from the outlet. We have shown that these phase shifts alone can cause constructive or destructive interference along the river link but that the physical parameters that determine the phase shift have a greater impact on the streamflow as it propagates downstream. The streamflows computed using different physical parameters demonstrate that the decreasing amplitude and increasing time delay in observed streamflows can be attributed to the decreasing velocity in the river network during dry conditions, and they are not necessarily due to soil-water processes, as was previously thought, which 
supports the hypothesis of Wondzell et al. (2007). Furthermore, the structure of the analytic solution indicates that the time delay increases continuously as the river network velocity continuously decreases, so that the time delay can be predictable depending on stream velocity. We apply the resulting solution to several locations in the Dry Creek Experimental Watershed using parameters determined by streamflow at the outlet. We then compare the streamflows resulting from our solution with observations. Our solution offers a good approximation for the streamflow at locations with larger upstream area (e.g., LG, C2M, and C2E), matching the magnitude of the streamflow and the amplitude and timing of the oscillations. Our results, however, do not disprove the hypothesis that delays can come from subsurface flow processes.
As a next step, we propose to test the analytic solutions herein in networks with different geomorphological structures in order to compare the resulting streamflow amplitudes and emphasize the dependence upon network geometry. We suggest subsequently comparing our analytic solutions with the numerical results obtained using nonlinear transport equations, which will demonstrate the relationship between link propagation at the hillslope scale and streamflow at the catchment scale. Careful field experiments would be necessary to provide a definitive conclusion about the attribution of time delays.

\section{Data availability}

Data available at https://earth.boisestate.edu/drycreek/data/. 
Appendix A: Development of streamflow solution for uniform $k$ value over all links in the river network

In order to simplify our calculations below, we will use the notation $\omega=2 \pi \nu$ and $\psi=2 \pi \nu \phi$.

We prove Eq. 7 from Sect. 3.1 by using the method of mathematical induction. The isolated effects of runoff from link $i$ on links downstream are found by applying the transport equation

$$
\frac{d q_{i}(t)}{\mathrm{d} t}=k\left(B e^{-A t}+C e^{-A t} \sin (\omega t-\psi)-q_{i}(t)\right)
$$

We did not include in Eq. (A1) any upstream links because we are trying to isolate the effects on streamflow due to runoff from hillslope $i$. Therefore, we treat it as an external link. Equation (A1) is a nonhomogeneous linear ordinary differential equation of the form

$\frac{d q_{i}}{\mathrm{~d} t}=k f_{i}(t)-k q_{i}$

and has the solution

$q_{i}(t)=q_{i}(0) e^{-k t}+k e^{-k t} \int_{0}^{t} f_{i}(s) e^{k s} \mathrm{~d} s$.

As we trace the runoff downstream, the function $f_{i}(t)$ is the input to the link, which can come from upstream sources or from runoff from the adjacent hillslope. Since link $i$ is arbitrary, we will consider it to be the first link in a path to the outlet, so it will be labeled link 1 , having flow $q_{1}$, and the next link downstream will be labeled link 2, etc. Since $f_{1}(t)$ consists only of baseflow, the solution $q_{1}$ according to Eq. (A3) becomes

$$
\begin{aligned}
q_{1}(t) & =q_{1}(0) e^{-k t}+k e^{-k t} \int_{0}^{t}\left[B e^{(k-A) s}\right. \\
& \left.+C e^{(k-A) s} \sin (\omega s-\psi)\right] \mathrm{d} s \\
& =q_{1}(0) e^{-k t}+B k e^{-k t}\left(\frac{e^{(k-A) t}}{k-A}-\frac{1}{k-A}\right) \\
& +C k e^{-k t} \int_{0}^{t} e^{(k-A) s} \sin (\omega s-\psi) \mathrm{d} s .
\end{aligned}
$$

The solution to the latter integral is

$$
\begin{aligned}
& \int_{0}^{t} e^{(k-A) s} \sin (\omega s-\psi) \mathrm{d} s=\frac{e^{(k-A) t}}{\sqrt{(k-A)^{2}+\omega^{2}}} \sin (\omega t-\psi-\varphi) \\
& +\frac{\sin (\psi+\varphi)}{\sqrt{(k-A)^{2}+\omega^{2}}},
\end{aligned}
$$

and $\varphi$ is defined by its sine and cosine functions:

$$
\sin (\varphi)=\frac{\omega}{\sqrt{(k-A)^{2}+\omega^{2}}},
$$

$\cos (\varphi)=\frac{k-A}{\sqrt{(k-A)^{2}+\omega^{2}}}$.

Substituting this integral back into Eq. (A4), we obtain

$$
\begin{aligned}
q_{1}(t) & =\left(q_{1}(0)-\frac{k}{k-A} B+\frac{k}{\sqrt{(k-A)^{2}+\omega^{2}}} C \sin (\psi+\varphi)\right) e^{-k t} \\
& +\left(\frac{k}{k-A} B+\frac{k}{\sqrt{(k-A)^{2}+\omega^{2}}} C \sin (\omega t-\psi-\varphi)\right) e^{-A t} .
\end{aligned}
$$

To find an algorithmic method to compute the coefficients of the solution $q_{n}(t)$ for $n \geq 1$, we define the following:

$$
\begin{array}{ll}
\mathcal{K}_{n}=C \prod_{j=1}^{n} \frac{k}{\sqrt{(k-A)^{2}+\omega^{2}}} & n \geq 1, \\
\mathcal{J}_{n}=B \prod_{j=1}^{n} \frac{k}{k-A} & n \geq 1, \\
\Phi_{n}=\psi+\sum_{j=1}^{n} \varphi & n \geq 1, \\
\mathcal{L}_{j}=q_{j}(0)-\mathcal{J}_{j}+\mathcal{K}_{j} \sin \left(\Phi_{j}\right) & j=1, \ldots, n .
\end{array}
$$

Using these newly defined quantities from Eqs. (A6), (A7), (A8), and (A9), the flow at the outlet of link 1 can be rewritten as

$q_{1}=\mathcal{L}_{1} e^{-k t}+e^{-A t}\left[\mathcal{J}_{1}+\mathcal{K}_{1} \sin \left(\omega t-\Phi_{1}\right)\right]$

To find the solution for the next link downstream (link 2), the flow from link 1, given by Eq. (A10), is included as $q_{\mathrm{in}_{1}}$ as the transport Eq. (2) is applied to link 2. Integration by parts will again be used to find the solution to

$\frac{d q_{2}}{\mathrm{~d} t}=k\left(q_{1}-q_{2}\right)$.

Using Eq. (A3),

$$
\begin{aligned}
q_{2}(t) & =q_{2}(0) e^{-k t}+k e^{-k t} \int_{0}^{t} q_{1}(s) e^{k s} \mathrm{~d} s \\
& =q_{2}(0) e^{-k t}+k e^{-k t} \mathcal{L}_{1} t+k e^{-k t} \mathcal{J}_{1}\left(\frac{e^{(k-A) t}}{k-A}-\frac{1}{k-A}\right) \\
& +k e^{-k t} \mathcal{K}_{1} \int_{0}^{t} e^{(k-A) s} \sin \left(\omega s-\Phi_{1}\right) \mathrm{d} s .
\end{aligned}
$$

The integral in Eq. (A11) is very similar to that in Eq. (A4), with the only differences being the argument of the sine function in the initial integral. After integration by parts, the equation for streamflow $q_{2}(t)$ becomes

$$
q_{2}=q_{2}(0) e^{-k t}+k e^{-k t} \mathcal{L}_{1} t+k e^{-k t} \mathcal{J}_{1}\left(\frac{e^{(k-A) t}}{k-A}-\frac{1}{k-A}\right)
$$


$+k e^{-k t} \mathcal{K}_{1}\left(\frac{1}{\sqrt{(k-A)^{2}+\omega^{2}}}\left(e^{(k-A) t} \sin \left(\omega t-\Phi_{2}\right)+\sin \left(\Phi_{2}\right)\right)\right)$

or, equivalently,

$q_{2}=\left[\mathcal{L}_{2}+k t \mathcal{L}_{1}\right] e^{-k t}+\left[\mathcal{J}_{2}+\mathcal{K}_{2} \sin \left(\omega t-\Phi_{2}\right)\right] e^{-A t}$.

By mathematical induction, using the same strategy for calculations along the path to the river network outlet, we can compute the contribution of runoff from any river link to flow at the outlet. For a given link that is at topological distance $n$ from the outlet (or an alternative location from which flow is observed), its contribution to the flow at the outlet is

$q_{n}(t)=e^{-A t}\left[\mathcal{J}_{n}+\mathcal{K}_{n} \sin \left(\omega t-\Phi_{n}\right)\right]+e^{-k t} \sum_{j=0}^{n-1} \mathcal{L}_{n-j} \frac{(k t)^{j}}{j !}$.

Given that $\omega=2 \pi \nu$ and using the notation $\varphi=2 \pi \nu \theta$, Eqs. (6), (7), (8), and (9) immediately will result.
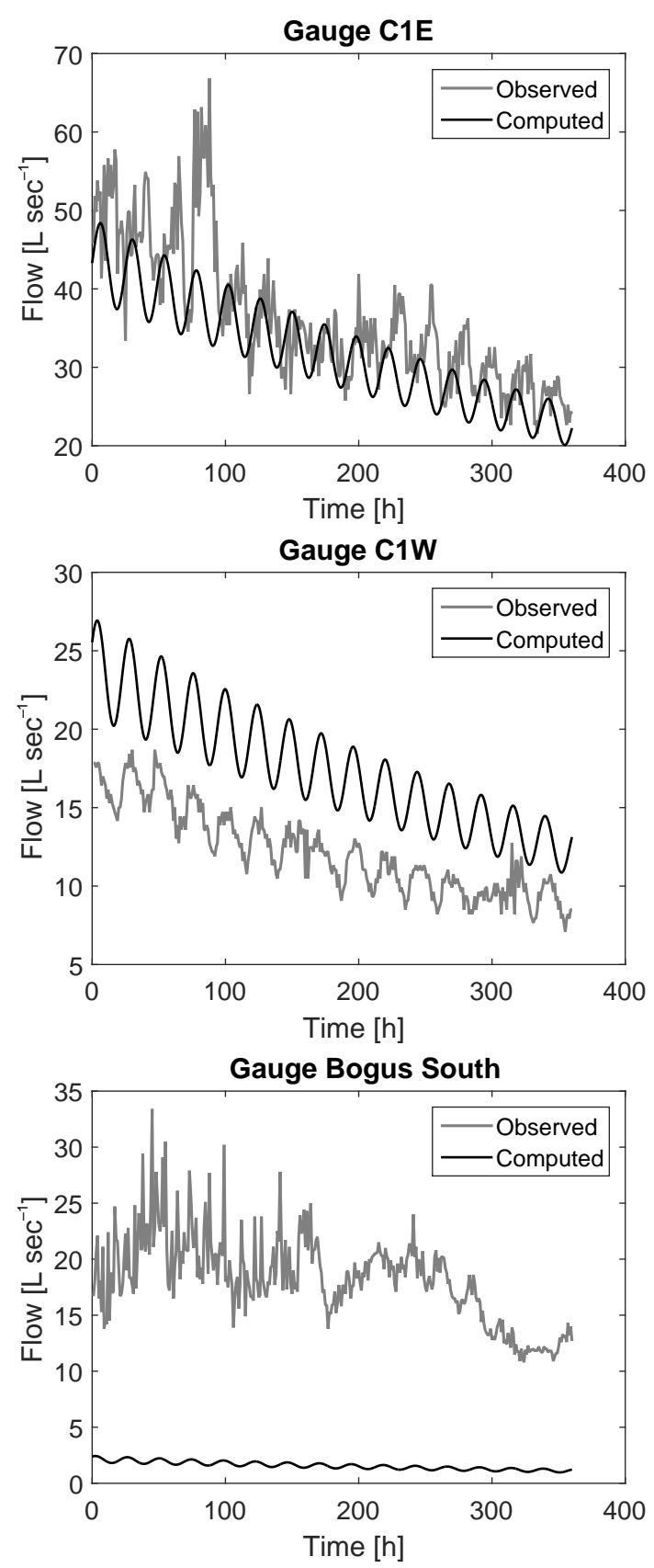

Figure B1. Observed streamflow and streamflow fitted using Eq. (16) at three upstream gauges in the Dry Creek Experimental Watershed: Con1East (top), Con1West (center), and Bogus South (bottom).

\section{Appendix B: Locations excluded from the analysis}

Because the Dry Creek Experimental Watershed includes stream gauges at seven different locations, we sought to compare our solution at all of these locations. One such gauge (called Treeline) did not experience streamflow during the duration of our observations. Three other gauges - Con1East, Con1West, and Bogus South (labeled C1E, C1W, and Bogus, 
respectively) - recorded streamflows which can be found in Fig. B1 along with our solution given by Eq. (16) at those locations.

As can be seen in Fig. B1, our solution does not offer a good fit to observed data at these three locations. Both $\mathrm{C} 1 \mathrm{E}$ and Bogus supply noisy signals, which do not have the obvious daily oscillations characteristic of the streamflows further downstream. The magnitude of the observed streamflow at Bogus is particularly interesting, because the area upstream of the gauge is $0.634 \mathrm{~km}^{2}$, and the average streamflow is around $18 \mathrm{~L} \mathrm{~s}^{-1}$. The streamflow at $\mathrm{C} 1 \mathrm{~W}$ is similar, with a magnitude of the average streamflow of about $13 \mathrm{~L} \mathrm{~s}^{-1}$, but the area upstream of $\mathrm{C} 1 \mathrm{~W}$ is $3.85 \mathrm{~km}^{2}$, so we should expect a significant difference between the streamflows at these two locations, and $\mathrm{C} 1 \mathrm{~W}$ should certainly experience larger values than Bogus. Because of this, we believe the observed streamflow at the Bogus site is unreliable.
The observed and predicted streamflow at the location C1E can be found in the left panel of Fig. B1. Again, the observations are especially noisy and have no apparent daily oscillations. However, our solution for streamflow has magnitude very close to observed values. We cannot conclude from this that our solution is incorrect, but it relied upon the assumption of smooth oscillatory runoff even at the hillslope scale. These noisy signals imply that the assumption is incorrect at some locations.

The center panel of Fig. B1 shows the observed and predicted streamflows at $\mathrm{C} 1 \mathrm{~W}$. Our solution does not offer a good fit to the data here in either the amplitude of the oscillations or the exact values of the streamflow. The values are of the same order of magnitude, however, and are reasonable for both $\mathrm{C} 1 \mathrm{E}$ and $\mathrm{C} 1 \mathrm{~W}$. 
Acknowledgements. This material is based on work supported by the National Science Foundation under grant number NSF DMS1025483 and financial support from the Iowa Flood Center. The authors also want to acknowledge Witold Krajewski from the University of Iowa and IIHR for helpful discussions and feedback during the preparation of the manuscript.

Edited by: T. Hengl

\section{References}

Arnell, N.: The effect of climate change on hydrological regimes in Europe: a continental perspective, Global Environ. Chang. 9 5-23, 1998.

Bond, B., Jones, J., Moore, G., Phillips, N., Post, D., and McDonnell, J.: The zone of vegetation influence on baseflow revealed by diel patterns of streamflow and vegetation water use in a headwater basin, Hydrol. Process., 16, 1671-1677, 2002.

Burn, D., Buttle, J., Caissie, D., MacCulloch, G., Spence, C., and Stahl, K.: The Processes, Patterns and Impacts of Low Flows Across Canada, Canad. Water Resour. J., 33 107-124, 2008.

Burt, T. P.: Diurnal variations in stream discharge and throughflow during a period of low flow, J. Hydrol., 41 291-301, 1979.

Graham, C., Barnard, H., Kavanagh, K., and McNamara, J.: Catchment scale controls the temporal connection of transpiration and diel fluctuations in streamflow, Hydrol. Process., 27 2541-2556, 2013.

Gribovszki, Z., Kalicz, P., Szilagyi, J., and Kucsara, M.: Riparian zone evapotranspiration estimation from diurnal groundwater level fluctuations, J. Hydrol., 349 6-17, 2008.

Gribovszki, Z., Szilagyi, J., and Kalicz, P. Diurnal fluctuations in shallow groundwater levels and streamflow rates and their interpretation - A review, J. Hydrol., 385, 371-383, 2010.
McNamara, J. and Aishlin, P.: Dry Creek Data, https://earth. boisestate.edu/drycreek/data/, 2016.

Mandelbrot, B. B. and Vicsek, T. Directed recursive models for fractal growth, J. Phys., 22, L377-L383, 1989.

Mantilla, R. and Gupta, V. K.: A GIS numerical framework to study the process basis of scaling statistics in river networks, Geosci. Remote Sens. Lett., 2.4 404-408, 2005.

Mantilla, R., Gupta, V., and Mesa, O.: Role of coupled flow dynamics and real network structures on Hortonian scaling of peak flows, J. Hydrol., 322, 155-167, 2006.

Mantilla, R., Gupta, V. K., and Troutman, B. M.: Scaling of peak flows with constant flow velocity in random selfsimilar networks, Nonlin. Processes Geophys., 18, 489-502, doi:10.5194/npg-18-489-2011, 2011.

McNamara, J. P.: Continuous monitoring in the Dry Creek Experimental WatershedHydrologic Sciences, Dept of Geoscience, Boise State University, Boise, ID, 2012.

Mul, M., Kemerink, J., Vyagusa, N., Mshana, M., van der Zaag, P., and Makurira, H.: Water allocation practices among smallholder farmers in the South Pare Mountains, Tanzania: The issue of scale, Agr. Water Manage., 98, 1752-1760, 2011.

Peckham, S.: New results for self-similar trees with applications to river networks, Water Resour. Res., 31, 1023-1029, 1995.

Rodriguez-Iturbe, I. and Rinaldo, A. Fractal river basins: chance and self-organization Cambridge University Press, 15-18, 2001.

Stott, R. E. and Burt, T. P.: Potassium chemistry of a small upland stream following a major drought, Hydrol. Process., 11, 189201, 1997.

Wondzell, S., Gooseff, M., and McGlynn, B.: Flow velocity and the hydrologic behavior of streams during baseflow, Geophys. Res. Lett., 34, L24404, doi:10.1029/2007GL031256, 2007.

Wondzell, S., Gooseff, M., and McGlynn, B.: An analysis of alternative conceptual models relating hyperheic exchange flow to diel fluctuations in discharge during baseflow recession, Hydrol. Process., 24, 686-694, 2010. 